\title{
Measurement of the conductance distribution function at a quantum Hall transition
}

\author{
D. H. Cobdent国 and E. Kogan ${ }^{\S}$ \\ ${ }^{\dagger}$ Cavendish Laboratory, Madingley Road, Cambridge CB3 0HE, United Kingdom \\ $\S$ Jack and Pearl Resnick Institute of Advanced Technology, \\ Department of Physics, Bar-Ilan University, Ramat-Gan 52900, Israel
}

(May 24, 2021)

\begin{abstract}
We study experimentally the reproducible conductance fluctuations between the quantum Hall plateaus in the conductance of two-terminal submicron silicon MOSFETs. For the dramatic fluctuations at the insulator-to-firstplateau transition we find a conductance distribution that is approximately uniform between zero and $e^{2} / h$. We point out that this is consistent with the prediction of random $S$-matrix theory for a conductor with single-channel leads in a magnetic field.
\end{abstract}

PACS numbers: 73.23.-b, 73.23.Ad, 73.40.Hm 
Reproducible fluctuations in the conductance of mesoscopic electronic devices have been a subject of continued interest for more than a decadel. At low magnetic fields they are now rather well understood in the case of diffusive conductors 8 , while their manifestation in chaotic ballistic cavities and quantum dots is under intensive study $\mathrm{B}^{4}$ at present. However, relatively little is known about the fluctuations that occur in high magnetic fields. For a two-dimensional system in which the quantum Hall effect dominates, the fluctuations occur whenever the conductivity is not quantizede日e 9 , i.e. in the transition regions, where the physics is tremendously subtle 10 .

At present, two complementary microscopic pictures exist of the cause of the fluctuations at high field. In one picture, transport near the center of the transition is assumed to be in the diffusive limit, with modifications to the diffusion equation to allow for Landau quantization and boundary effects 112 . In this case the fluctuations resemble those at zero magnetic field $B$, but with correlation scales that vary with $B$, as has been observed in experiment, 14 , 目. This picture does not take into acount localization (and therefore the quantum Hall effect), and is applicable only when the index of the highest occupied Landau level is large. In the other picture, transport is in the adiabatic limit, and peaks in the conductance or resistance arise from resonant tunneling between edge states through localized bulk states]. This picture is applicable if the disorder potential is smooth on the scale of the magnetic length, so that edge states are well defined within the sample. It has been used to interpret fluctuations at the edges of quantum Hall zeros in the four-terminal longitudinal resistance of high mobility GaAs/AlGaAs heterostructure devices 1 . However, one is faced with the problem that in the archetypal integer quantum Hall system, the silicon MOSFET (metaloxide-silicon field-effect transistor), which we study here, the conditions are such that neither of these two limiting cases pertains. In MOSFETs the disorder is very short range, while wide integer quantum Hall plateaus are seen at low Landau-level index. This, combined with the unknown but certainly important consequences of interactions, makes it very difficult to interpret the dramatic fluctuations we observe in our devices in terms of a microscopic model at present. 
Nonetheless, the techniques of random matrix theory (RMT) allow one to make predictions for the statistical properties of conductance fluctuations without knowing all the microscopic details. RMT has recently been successfully applied to diffusive transport in small metallic objects and ballistic transport in chaotic cavities16. Here we make the suggestion that under certain conditions RMT may also be applied to transport at quantum Hall transitions, as described by the scattering matrix between incoming and outgoing edge channels 1 . We present experimental evidence in support of this, namely that the measured distribution of the fluctuations on a quantum Hall transition is approximately flat between zero and $e^{2} / h$. The same flat distribution is predicted by RMT for the single-channel $S$ matrix at high $B$. 18 . In contrast, at low $B$ we observe the Gaussian distribution expected when the conductance (and therefore the number of channels) is large 2 .

The devices used are industrial process two-terminal silicon MOSFETs with oxide thickness $250 \AA$ and a range of effective channel dimensions down to $0.4 \mu \mathrm{m}$. The rough sketch in the inset to Figure 1 emphasizes their essentially very simple geometry. The $\mathrm{n}+$ contacts, indicated in the plan view by rectangles with diagonal crosses, are self-aligned to the gate. Each device can be thought of as a rectangle of two-dimensional electron gas with an almost ideal metallic contact at each end and a variable carrier density controlled linearly by the gate voltage $V_{g}$. The conductance was measured in a dilution refrigerator using a low-frequency ac voltage of $10 \mu \mathrm{V}$ (comparable with the effective electron temperature of approximately $100 \mathrm{mK}$ ), a virtual-earth current preamplifier and a lock-in amplifier. The low temperature mobility was around $2000 \mathrm{~cm}^{2} \mathrm{~V}^{-1} \mathrm{~s}^{-1}$, corresponding to a mean free path of about $200 \AA$. At base temperature the phase coherence length was around $0.4 \mu \mathrm{m}$, as deduced from the low- $B$ fluctuations (see below) 19 .

The characteristics of a $0.6 \times 0.6 \mu \mathrm{m}^{2}$ device are shown in Figure 1(a) at $B=0$ and Figure $1(\mathrm{~b})$ at $B=16 \mathrm{~T}$. At $B=0$ the conductance $G$ as a function of $V_{g}$ shows universal fluctuations superimposed on a rising background above a threshold of about $2.2 \mathrm{~V}$ (this threshold varies randomly between devices over a range of $500 \mathrm{mV}$ or so). At $16 \mathrm{~T}$ the situation is very different: the conductance increases through a series of quantum Hall 
plateaus. This is a consequence of the fact that for such a nearly ideal two-terminal device the Landauer formula (Eq. (2) below) applies directly to the measured conductance. On the plateaus, the conductance (in units of $e^{2} / h$ ) equals the number of transmitted edge chan-

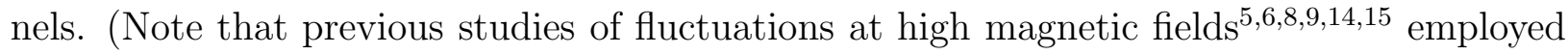
multi-terminal Hall-bar geometries for which the relation of the measured quantities to the transmission coefficients is less direct). Subtraction of a constant series resistance was found to bring all the plateaus very close to exact multiples of $e^{2} / h$. Because of the double spin and valley degeneracies at zero field, the first four plateaus are all associated with the lowest Landau level. On the transitions between adjacent plateaus there are rapid, reproducible fluctuations.

Before discussing these fluctuations at high $B$ further, let us compare those at low $B$ with the existing standard theoryt. Below about $4 \mathrm{~T}$, sweeping $B$ generates fluctuations, as shown in Figure 2(a), which are statistically independent of those produced by sweeping $V_{g}$ (as in Figure 1(a)). The magnetic field correlation length 20 is $B_{c} \approx 30 \mathrm{mT}$, implying that the phase-coherent area is $h /\left(e B_{c}\right) \approx 0.14 \mu \mathrm{m}^{2}$, which is more than a third of the channel area, $0.36 \mu \mathrm{m}^{2}$. To obtain the conductance distribution function, similar $B$-sweeps were taken at a series of closely spaced values of $V_{g}$ between 3.6 and $4.0 \mathrm{~V}$ and a smooth monotonic background was subtracted from the $G-B-V_{g}$ dataset. The histogram of the resulting conductance values, offset by the average, $\bar{G}=17.5 e^{2} / h$, of $G$ over the entire dataset, is shown in Figure 2(b). Since $\bar{G} \gg 1$, a purely Gaussian distribution is expected 1 for a phase-coherent sample. The solid curve in the figure is the best fit Gaussian. Of course, for a highly phase-incoherent sample, effectively consisting of a large number of uncorrelated phase-coherent units, the central limit theorem will enforce a Gaussian distribution. However, there are fewer than three phase-coherent sub-units in this device, and we believe that the accurate measured Gaussianity reflects the intrinsic conductance distribution. The standard deviation of the measured distribution is $0.22 e^{2} / h$, which taking into account partial phase-breaking, uncertain geometrical factors, and an unknown contact resistance, is quite consistent with the measured value of $B_{c}$, according to the theory of universal conductance 
fluctuations 12 .

We now turn to the high magnetic field results. Figure 2(c) shows the lowest quantum Hall transition from Figure 1(b). The thicker solid line superimposed is the same data smoothed by averaging over $16 \mathrm{mV}$ in $V_{g}$. At the edges of the transition the fluctuations in $G$ resemble the resonant peaks and dips of the edge-state tunneling picture 7 ? . However, over a wide region of $V_{g}$, indicated by plotting the data using a thin solid line rather than a dashed line, $G$ fluctuates strongly between strict limits of zero and $e^{2} / h$. Throughout this region the smoothed conductance lies in the range $0.5 \pm 0.1 e^{2} / h$. Figure $2(\mathrm{~d})$ shows the distribution of conductance values for this region only. It is fairly uniform for $0<G<e^{2} / h$ and zero for $G>e^{2} / h$. This contrasts sharply with the strongly peaked, long-tailed Gaussian distribution for $B=0$ in Figure 2(b). For reasons which will be discussed below we have no way of improving the statistics here. However, we can estimate the error in the value for each bin in the histogram to be of the order of the variation from bin to bin. To within this error the distribution is flat between 0.1 and $0.9 e^{2} / h$.

Two versions of RMT have been used in transport theory. The first, that of random multiplicative transfer matrices 16 , can describe diffusive transport in quasi-one-dimensional systems. The second, that of the random $S$-matrix, is most appropriate for ballistic transport. Here we are dealing with a third kind of transport, which we imagine involves the adiabatic motion of electrons in rather long, percolating edge-like quantum Hall states. Taking into account also the strong disorder in the system, one might guess that this problem belongs in the same universality class as diffusive transport. However, the main aim of this paper is to point out that a flat distribution of the conductance between zero and $e^{2} / h$, compatible with what we observe for the first quantum Hall transition, is just what one gets from the simplest random $S$-matrix theory.

The appropriate RMT result has already been obtained in the analysis of chaotic ballistic

cavities 17,18 . For a sample connected between two leads each with $N$ channels, the $S$-matrix is 


$$
S=\left(\begin{array}{ll}
r & t^{\prime} \\
t & r^{\prime}
\end{array}\right),
$$

where $r$ and $t$ are the $N \times N$ reflection and transmission matrices for electrons incident from the left, and $r^{\prime}$ and $t^{\prime}$ are for those from right. Current conservation forces $S$ to be unitary, and since the strong magnetic field breaks time reversal symmetry the unitary ensemble, with symmetry index $\beta=2$, is appropriate. The two-terminal conductance is given by the Landauer formula,

$$
G=\left(e^{2} / h\right) \operatorname{Tr}\left\{t t^{\dagger}\right\}
$$

For a single quantum Hall transition, and especially the first (between $G=0$ and $e^{2} / h$ ), it is natural to take $N=1$. The $2 \times 2 S$-matrix then consists of the reflection and transmission coefficients for the lowest (spin-down, valley-one) edge state incident on the 2D region from the contacts. If $S$ is characterized by Dyson's circular ensemble then the conductance distribution function is given by 17.18

$$
P(G)=(\beta / 2) G^{-1+\beta / 2}
$$

For $\beta=2$, Eq. (3) gives a flat distribution between zero and $e^{2} / h$, with $\bar{G}=0.5 e^{2} / h$. The data in Fig. 2 (c) are particularly appropriate for comparison with this theory because of the wide range of $V_{g}$ over which the average of $G$ (the thick solid line) is close to $0.5 e^{2} / h$.

We emphasize that the form of the fluctuations on quantum Hall transitions is variable, and it is difficult to find instances like the one in Figure 2(b) where the data are suitable for obtaining a distribution function. This is illustrated by the examples of transitions shown in Figure 3. One inhibiting factor is that the correlation length, or period, of the fluctuations is usually longer than in this device. The period, and hence the number of peaks and dips, can in fact differ markedly between devices, as illustrated by trace (iii) from device 2, which is nominally identical to device 1 . A difference in period and amplitude may be explained by variations in the electron temperature, due to changes in the amount of heating by external electrical noise. However, temperature variations cannot account for 
the reproducible differences in period often seen between transitions in the same device, and even across a single transition, such as in trace (i). The monotonic increase in $\bar{G}$ and the uniformity of the fluctuations actually appear to be restricted to square devices and to the first quantum Hall transition only. For higher transitions, as illustrated by traces (i) and (ii) which are again from device 1, the potential for intervalley and spin-flip scattering may underlie the complex nonmonotonic behavior. Meanwhile, when the length/width ratio differs from unity, as it does for device 3 (trace (iv)), one can easily imagine the asymmetry between the average forward- and backward-scattering probabilities resulting in a situation where the $S$-matrix is not completely random 3 .

Nevertheless, we can discern the following patterns. First, as the device area increases, the period in $V_{g}$ of the fluctuations tends to decrease. Second, in devices where the length/width ratio is much less than or greater than unity, the valley-split plateaus are destroyed by fluctuations with respectively an enhanced or a reduced mean conductance, while the other plateaus are remarkably robust. Third, the fluctuations evolve in a surprisingly simple way with $B^{21}$. These results will be reported in detail elsewhere.

In summary, we have presented an experimental measurement of the conductance distribution of mesoscopic fluctuations at a quantum Hall transition. The results are consistent with the hypothesis that the distribution function is given by random matrix theory for the single-channel unitary $S$-matrix.

DHC is particularly grateful to Crispin Barnes for many stimulating discussions about the possible microscopic nature of the fluctuations. We also thank P. Mello and M. Pepper for helpful discussions, J. T. Nicholls for invaluable experimental assistance, and Yuki Oowaki of Toshiba for supplying the devices. DHC acknowledges the UK EPSRC for financial support. 


\section{REFERENCES}

* Presently at the Lawrence Berkeley Laboratory, Mail Stop 2/200, Berkeley, CA 94720.

${ }^{1}$ For reviews of mesoscopic physics see C. W. J. Beenakker and H. van Houten, in Solid State Physics, edited by H. Ehrenreich and D. Turnbull (Academic Press, New York, 1991) Vol. 44, pp. 1-228; Mesoscopic Phenomena in Solids, edited by B. L. Altshuler, P. A. Lee, and R. A. Webb (North-Holland, New York, 1991).

${ }^{2}$ B. L. Altshuler, V. E. Kravtsov and I. V. Lerner, in Mesoscopic phenomena in solids, edited by B. L. Altshuler, P. A. Lee and R. A. Webb (North-Holland, Amsterdam, 1991).

${ }^{3}$ P. A. Mello, in Mesoscopic Quantum Physics, Les Houches, Session LXI, 1994, edited by E. Akkermans, G. Montambaux, J.-L. Pichard and J. Zinn-Justin, Elsevier Science, 1995; H. U. Baranger and P. A. Mello, Europhys. Lett. 33, 456 (1996).

${ }^{4}$ P. W. Brouwer and C. W. J. Beenakker, Phys. Rev. B 51, 7739 (1995); P. W. Brouwer, Phys. Rev. B 51, 16878 (1995).

${ }^{5}$ G. Timp, A. M. Chang, P. Mankiewich, R. Behringer, J. E. Cunningham, T. Y. Chang, and R.E. Howard, Phys. Rev. Lett. 59, 732 (1987).

${ }^{6}$ C. J. B. Ford, T. J. Thornton, R. Newbury, M. Pepper, H. Ahmed, D. C. Peacock, D. A. Ritchie, J. E. F. Frost, and G. A. C. Jones, Phys. Rev. B 38, 8518 (1988).

${ }^{7}$ J. K. Jain and S. A. Kivelson, Phys. Rev. Lett. 60, 1542 (1988).

8 J. A. Simmons, H. P. Wei, L. W. Engel, D. C. Tsui, and M. Shayegan, Phys. Rev. Lett. 63, 1731 (1989); J. A. Simmons, S. W. Hwang, D. C. Tsui, H. P. Wei, L. W. Engel, and M. Shayegan, Phys. Rev. B 44, 12933 (1991).

${ }^{9}$ P. C. Main, A. K. Geim, H. A. Carmona, C. V. Brown, T. J. Foster, R. Taboryski, and P. E. Lindelof, Phys. Rev. B 50, 4450 (1994).

${ }^{10}$ For a review see, e.g., The Quantum Hall Effect, edited by R. E. Prange and S. M. Girvin, 
Springer-Verlag, New York, 1990.

${ }^{11}$ Shanhui Xiong and A. Douglas Stone, Phys. Rev. Lett. 68, 3757 (1992).

${ }^{12}$ D. L. Maslov and Daniel Loss, Phys. Rev. Lett. 71, 4222 (1993).

${ }^{13}$ D. E. Khmelnitskii and M. Yosefin, Surf. Sci. 305, 507 (1994).

${ }^{14}$ A. K. Geim, P. C. Main, P. H. Beton, and L. Eaves, Phys. Rev. Lett. 69, 1248 (1992); C. V. Brown, A. K. Geim, T. J. Foster, C. J. G. M. Langerak, and P. C. Main, Phys. Rev. B 47, 10935 (1993).

${ }^{15}$ A. Morgan, D. H. Cobden, M. Pepper, G. Jin, Y. S. Tang, and C. D. W. Wilkinson, Phys. Rev. B 50, 12187 (1994).

${ }^{16}$ A. D. Stone, P. A. Mello, K. Muttalib, and J.-L. Pichard, in Mesoscopic Phenomena in Solids (Ref. [1]).

${ }^{17}$ Harold U. Baranger and Pier A. Mello, Phys. Rev. Lett. 73, 142 (1994).

${ }^{18}$ R. A. Jalabert, J.-L. Pichard, and C. W. J. Beenakker, Europhys. Lett. 27, 255 (1994).

${ }^{19}$ At magnetic fields below about $10 \mathrm{mT}$ the aluminium contacts went superconducting, making determination of the phase coherence length using weak localization studies impracticable. Measurements nominally at $B=0$ were actually done at $B=20 \mathrm{mT}$.

${ }^{20}$ B. I. Altshuler, JETP Lett. 41, 648 (1985); P. A. Lee and A. D. Stone, Phys. Rev. Lett. $55,1622(1985)$.

${ }^{21}$ The evolution with $B$ is so slow that it is not possible to significantly decrease the error in the measured distribution by combining datasets taken at different magnetic fields. 


\section{FIGURES}

FIG. 1. Characteristic of a $0.6 \times 0.6 \mu \mathrm{m}^{2}$ MOSFET device at $T \sim 100 \mathrm{mK}$, at (a) $B=0$ and (b) $B=16$ T. A $2.7 \mathrm{k} \Omega$ series resistance was subtracted from the data in (b). Inset: idealized sketches of a device in cross-section and plan view.

FIG. 2. (a) Sample of the universal conductance fluctuations at low magnetic field. (b) Histogram obtained from many similar datasets at different $V_{g}$, normalized to a maximum value of unity. The solid curve is the best Gaussian fit. (c) The first quantum Hall transition from Figure 1 (b). The thick solid line is the same data smoothed over $16 \mathrm{mV}$ in $V_{g}$. (d) Histogram of the region of datapoints joined with a thin solid line in (c), normalized to an average value of unity.

FIG. 3. Further examples of quantum Hall transitions at $B=16 \mathrm{~T}$. Each has been offset arbitrarily in $V_{g}$, for convenient comparison, but not in $G$. The device dimensions, in the form length $\times$ width, are indicated in microns. 


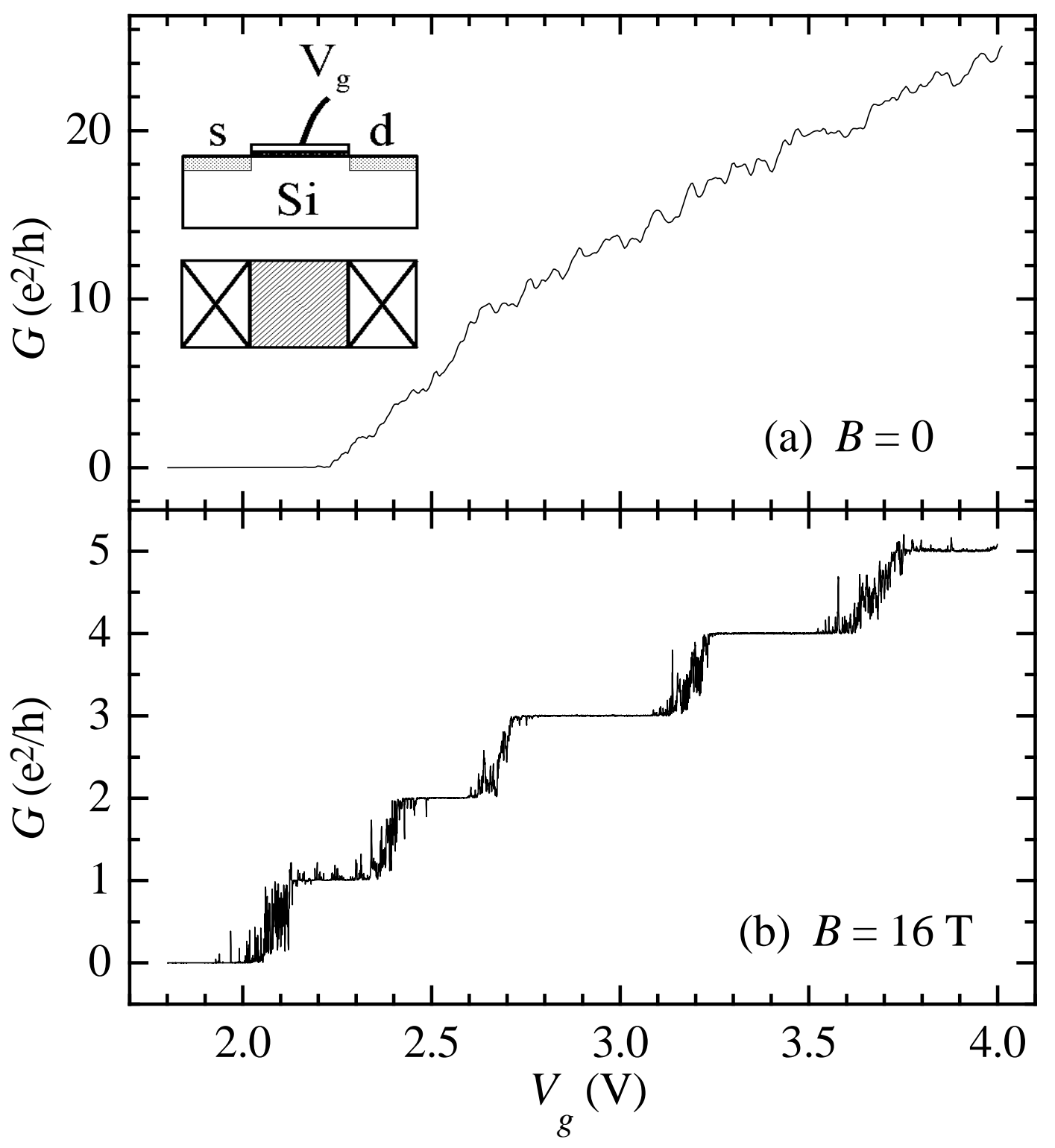



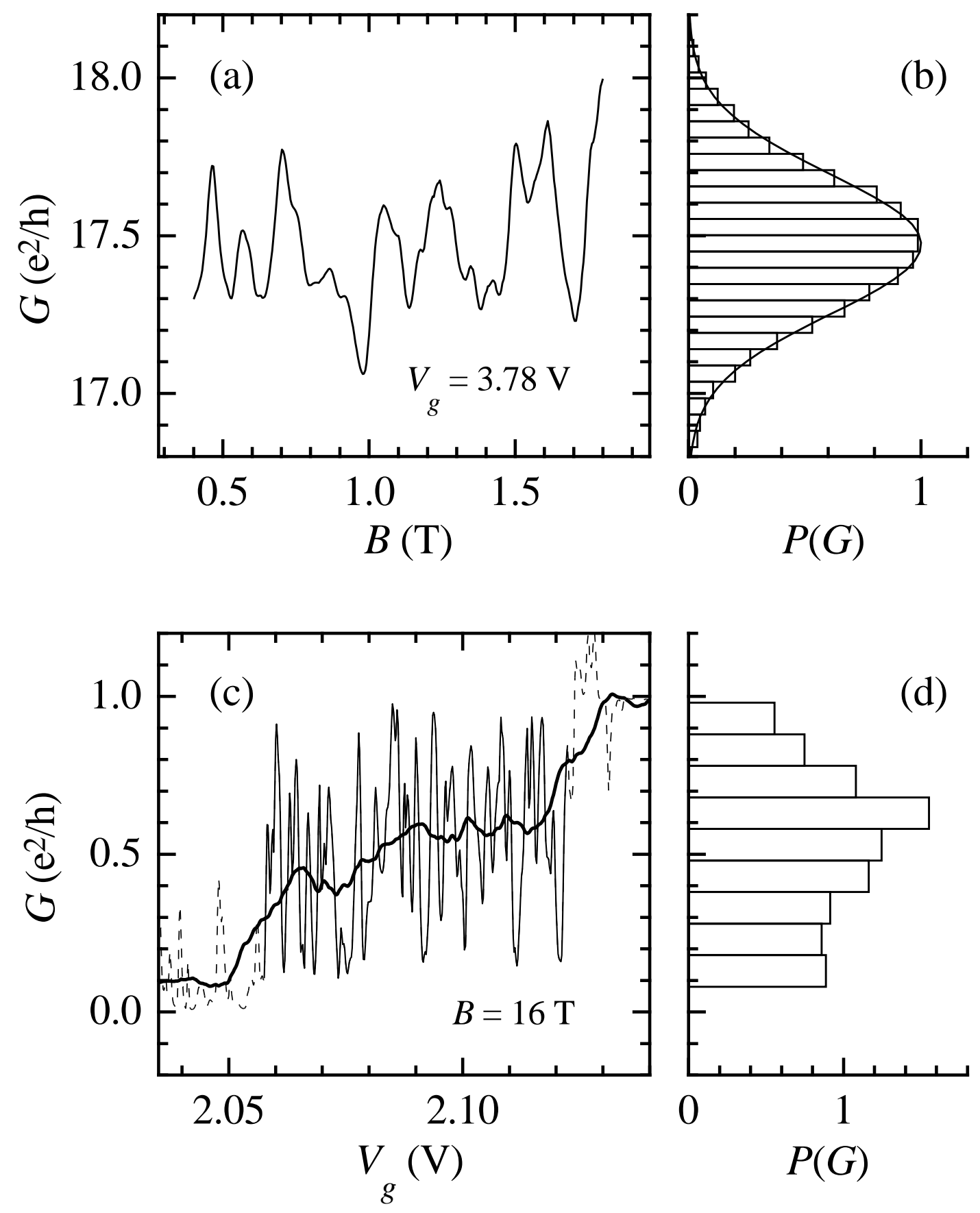


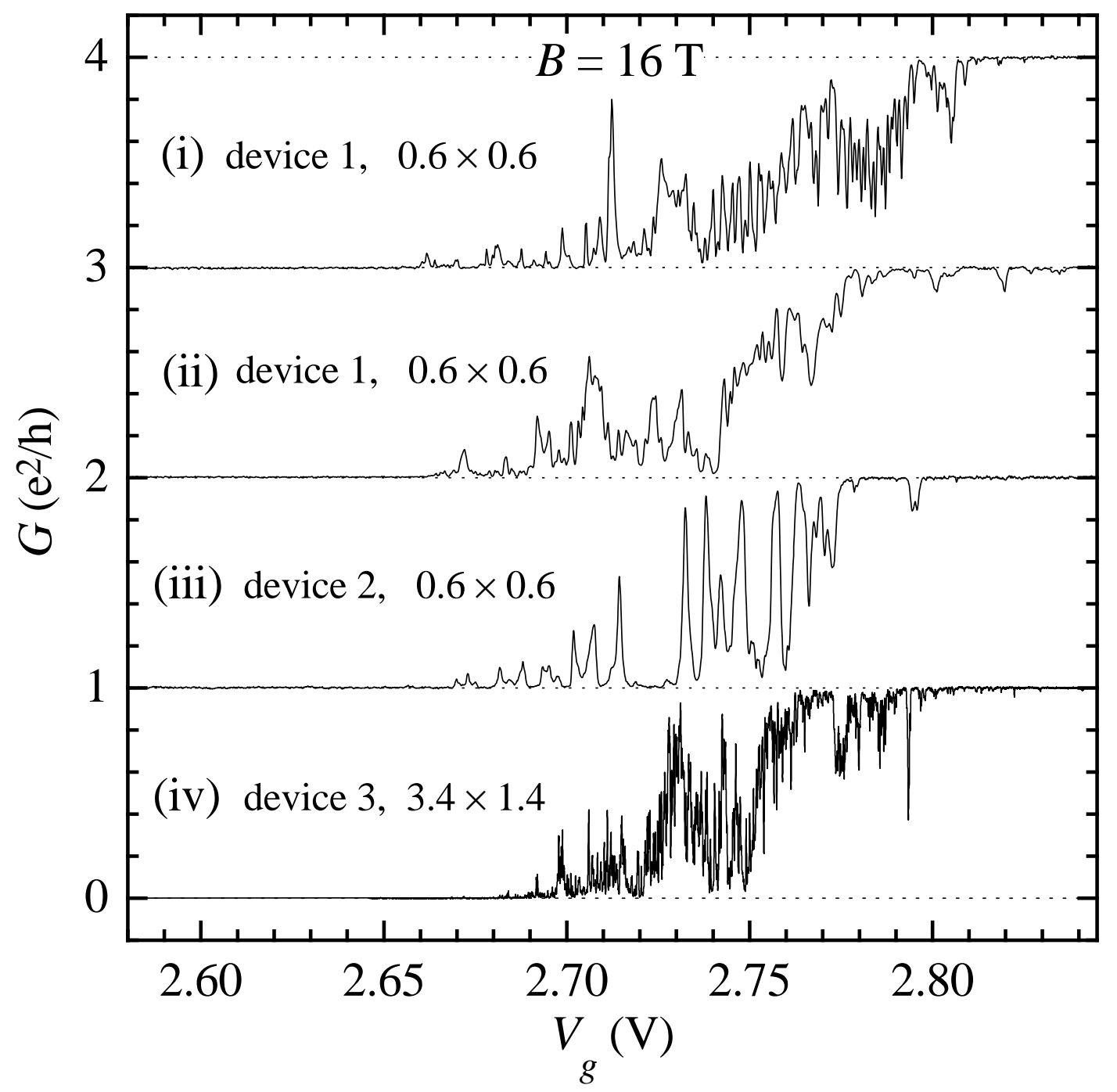

